\title{
Enquête sur les pratiques du spermogramme en France
}

\author{
Clavert A.*, Bourguignat A.**, Siest J.P. ${ }^{\circ}$, Ferard G. ${ }^{* *}$
}

* Laboratoire de Biologie de la Reproduction, Hôpitaux Universitaires de Strasbourg, BP 426, 67091 Strasbourg Cedex ; **Laboratoire de Biochimie appliquée, Faculté de Pharmacie, BP 24, 67401 Illkirch Cedex; 'Secrétariat technique des contrôles de qualité du CRECQ, 30 rue Lionnois, BP 292, 54005 Nancy.

\section{RESUME}

Un questionnaire détaillé à été envoyé à de nombreux laboratoires d'analyses médicales privés comme hospitaliers. Nous avons obtenu 214 réponses. La majorité des laboratoires participant à cette enquête réalisent moins de 5 spermogrammes par semaine, seuls 74 laboratoires ont une activité supérieure et peuvent être considérés comme spécialisés.

Parmi les différents items proposés, ceux portant sur la conservation du sperme, l'évaluation de la viscosité, la numération, l'évaluation de la mobilité, le spermocytogramme et l'étude des cellules rondes, révèlent une grande disparité des méthodologies.

L'analyse de ces variations met de mettre en évidence des divergences importantes dans des pratiques déterminantes pour la puissance diagnostique du spermogramme.

Le groupe de travail demande la mise en oeuvre de pratiques simples à réaliser de manière à se rapprocher des recommandations de l'O.M.S. : travailler à $37^{\circ} \mathrm{C}$, utiliser les critères de l'O.M.S. pour évaluation de la mobilité et du degré de tératospermie: un format commun de compte-rendu des résultats, élaboré par le groupe de travail, est proposé pour compléter cette démarche.

Mots clés : Spermogramme; Pratiques ; Procédures.

Un questionnaire élaboré par le groupe de travail "Assurance de Qualité en Biologie de la Reproduction" a été distribué aux membres des sociétés : BLEFCO, CECOS, SALF et SFBC. Nous remercions les secrétariats de ces sociétés de leur aide précieuse.

Sur les 2500 questionnaires ainsi distribués, nous avons reçu 214 réponses. Il est difficile de savoir combien de laboratoires ont été effectivement contactés puisque plusieurs membres d'un laboratoire peuvent appartenir à une même société et certains biologistes peuvent être membres de plusieurs sociétés. En outre, sont membres de la SFBC des laboratoires d'analyses médicales qui ne réalisent pas d'analyses de biologie de la reproduction.

D'après les agréments donnés par le Ministère de la Santé on compte en France : 23 centres pratiquant le don de sperme, 89 centres pratiquant la fécondation in vitro et 96 laboratoires autorisés à préparer $d u$ sperme en vue d'inséminations, soit au total 208 laboratoires, au maximum, ayant un agrément. 
La première question concernait le niveau d'activité du laboratoire. Elle a été évaluée en fonction du nombre de spermogrammes réalisés par semaine (Figure 1). Ainsi, nous avons été surpris de constater que la majorité des laboratoires ayant répondu réalisent moins de 5 spermogrammes par semaine ; seuls 74 ont une activité de plus de 5 spermogrammes. Le nombre réduit de laboratoires spécialisés ayant accepté de répondre au questionnaire montre que l'assurance de qualité n'est pas encore une priorité dans notre spécialité. Un exemple : seulement 13 CECOS sur 22 nous ont renvoyé le document, pourtant ces centres ont une pratique régulière du contrôle de qualité.

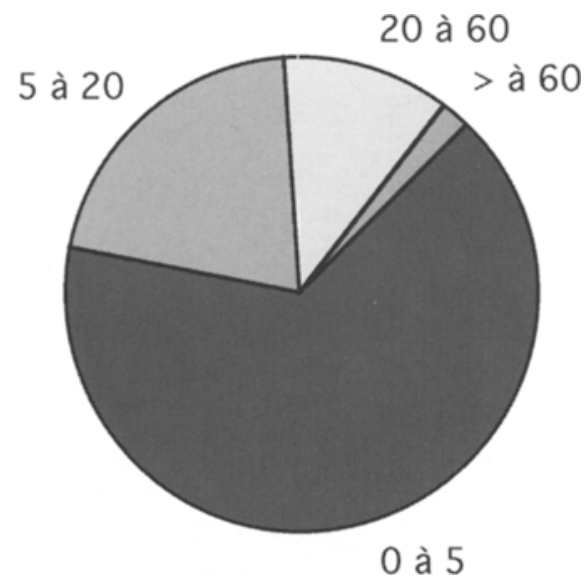

Figure 1 : Répartition des laboratoires ayant participé à l'enquête en fonction du nombre de spermogrammes réalisés par semaine.

\section{LE QUESTIONNAIRE}

L'esprit, dans lequel ce questionnaire a été préparé, était de pouvoir recenser les différentes pratiques de la totalité de la chaîne des événements, de l'arrivée du patient au laboratoire, à l'expédition des résultats. Ce questionnaire était à la fois précis et laissait des espaces pour des réponses ouvertes. En outre, il était demandé de joindre dans la mesure du possible des documents (grille d'analyse et réponse type). Ces documents joints nous ont per- mis de constater que sous un intitulé identique existaient de nombreuses variantes et que, dans certains cas, l'homogénéité n'était qu'apparente.

Les différents points techniques abordés étaient :

- précautions avant prélèvement

- recueil et réceptacles

- liquéfaction

- conditions de conservation du sperme

- mesure du volume

- évaluation de la viscosité

- numération des spermatozoïdes

- évaluation de la mobilité

- spermocytogramme

- étude des cellules rondes

\section{RESULTATS}

Les items concernant l'hygiène de prélèvement et l'évaluation du volume et de la viscosité ne mettent pas en évidence de grosses divergences ; par contre, la conservation du sperme, la liquéfaction, la numération, l'évaluation de la mobilité, le spermocytogramme et l'étude des cellules rondes, révèlent une grande diversité méthodologique.

\section{Conservation du sperme}

Sur les 214 laboratoires ayant répondu seuls 140 conservent le sperme à l'étuve à $37^{\circ} \mathrm{C}$, les autres laboratoires conservent le sperme à température ambiante.

\section{Evaluation du temps de liquéfaction et de la viscosité}

Ce temps dépendant de la température, il n'est pas possible de comparer les résultats si la conservation de l'éjaculat ne se fait pas à température normalisée.

Parmi les 140 laboratoires travaillant à $37^{\circ}$ C, 84 évaluent la viscosité à 30 minutes et 56 après $20 \mathrm{~min}$. 


\section{Numération}

Le type de cellule choisi est très varié, 78 utilisent une cellule de Malassez, 58 une cellule de Thoma, 30 une cellule de Makler, mais les cellules de Kova, Neubauer et Cyturine sont également utilisées.

Si la cellule de Makler ne nécessite pas de dilution, pour les autre cellules, 74 diluent au $1 / 10$ e ou $1 / 20$ e alors que 42 équipes adaptent la dilution à la concentration estimée du sperme.

103 laboratoires lisent une seule grille, 74 lisent deux grilles et font la moyenne.

\section{Mobilité}

Sur les 214 laboratoires ayant répondu seuls 34 évaluent la mobilité de manière objective par CASA, 18 utilisent un appareil Hamilton, 8 par un ATS 40 et 1 par un Cell Track.

La lecture subjective se fait pour 70 laboratoires au microscope à contraste de phase et 122 laboratoires disent utiliser un microscope à transmission.

Le grossissement est très variable, 55 utilisent un faible grossissement ( 10 ou $25 \mathrm{X}$ ), 109 utilisent le $40 \mathrm{X}$ et 2 laboratoires le $100 \mathrm{X}$.

La préparation de l'échantillon présente également de grosses variations : 76 laboratoires calibrent leur goutte en fonction de la taille de la lamelle et 89 laboratoires travaillent à une temprérature de $37^{\circ} \mathrm{C}$.

Le nombre de lectures est : pour 10 laboratoires de 1 fois à 1 heure, pour 78 de 2 fois ( 1 et $4 \mathrm{~h}$ ), pour 43 de 3 fois (1, 3 et $6 \mathrm{~h}$ ) et 30 laboratoires font plus de 3 lectures.

\section{Spermocytogramme}

La coloration est pour 61 laboratoires le Schorr, 58 le M.G.G., 35 le Papanicolaou, 8 le Test simplet (Boehringer) et 19 utilisent des colorations diverses.

L'observation est faite pour 180 laboratoires au 100 à immersion et 34 utilisent un grossissement de $40 \mathrm{X}$ et moins.

La grille de lecture utilisée est variable à plusieurs points.

104 laboratoires se réfèrent à la grille de David, 24 à celle de l'OMS, 4 à celle de Kruger et 4 à des grilles simplifiées ; mais l'étude des documents joints montre que l'intitulé : grille de David cache une grande hétérogénéité. En effet les grilles que nous avons pu étudier étaient pour partie des grilles correspondant à celles proposées par David en 1974 mais d'autres correspondaient à des améliorations apportées progressivement par l'équipe David puis par l'équipe Jouannet.

L'I.A.M. (Indice d'Anomalies Multiples) est calculé par 95 laboratoires sur les 214.

\section{Etude des cellules rondes}

Si les cellules rondes sont régulièrement comptées lors de la numération des spermatozoïdes, seuls 54 laboratoires réalisent une mise en évidence de la peroxydase pour caractériser les polynucléaires.

162 laboratoires recherchent les polynucléaires sur les frottis et 134 d'entre eux étudient la morphologie de l'ensemble des cellules rondes.

\section{DISCUSSION}

Une première remarque s'impose : il existe une très grande dispersion dans les pratiques.

Elles ont besoin d'être normalisées pour éviter de trop importantes dispersions dans les résultats d'une analyse qui s'avère dans la pratique des plus importantes pour les étapes ultérieures de l'exploration d'une stérilité conjugale.

\section{Température de conservation de l'éjaculat et temps de liquéfaction}

Une première proposition est de conserver le sperme et de réaliser les différentes ana- 
lyses à $37{ }^{\circ} \mathrm{C}$. Ce paramètre joue un rôle important sur la vitesse de liquéfaction, la mobilité et la survie des spermatozoîdes. L'utilisation d'une étuve et d'une platine microscopique chauffante permet facilement de maintenir le sperme à cette température pendant toutes les manipulations.

La lecture de la viscosité après 30 minutes d'incubation à $37{ }^{\circ} \mathrm{C}$ semble être la condition standard pour évaluer le processus de liquéfaction.

\section{Numération}

L'utilisation de différentes cellules peut être un facteur de variation. Une étude comparative entre la cellule de Makler et les différents hémocytomètres doit être entreprise pour évaluer une éventuelle discordance des résultats.

En outre,comme le préconise le manuel de l'OMS, la dilution du sperme avant numération doit être adaptée à la concentration évaluée lors de la première analyse de la mobilité.

La lecture de deux grilles par dilution permet de vérifier l'homogénéité de la dilution. Lorsqu'il existe une différence trop importante entre les deux numérations, une nouvelle dilution doit être réalisée.

\section{Evaluation de la mobilité}

En dehors de l'utilisation d'analyseur d'images, l'évaluation de la mobilité est subjective et dépend en grande partie des critères de l'observateur. Avant de vouloir essayer de normaliser ces critères subjectifs, il est impératif que les conditions d'observation soient strictement définies.

- L'utilisation d'un microscope à contraste de phase avec une platine chauffante et équipé d'objectifs $10 \mathrm{x}, 20$ ou $25 \mathrm{x}$ et éventuellement $40 \mathrm{x}$ est indispensable pour bien observer les spermatozoïdes mobiles et immobiles, l'utilisation d'un microscope à transmission surestime le nombre de spermatozoïdes mobiles.
- Une goutte calibrée $10 \mu \mathrm{l}$ pour une lamelle $22 \times 22 \mathrm{~mm}$ : cette précaution permet d'observer les spermatozoïdes dans des conditions reproductibles. Dans ces conditions l'épaisseur est suffisante pour permettre au mouvement du spermatozoïde de se dérouler normalement.

Les mouvements doivent être classés suivant les critères de l'OMS.
A- rapide et progressif
B- lent ou faiblement progressif
$\mathrm{C}$ - mobile mais non progressif
D- immobile

Les mouvements hyperactivés doivent être signalés séparément.

\section{Spermocytogramme}

Le manuel de l'OMS préconise la coloration de Schorr ou de Papanicolaou.

Des contrôles de qualité ont démontré que l'utilisation d'autres colorations que celles recommandées, augmente le nombre de spermatozoïdes normaux ; une analyse plus fine a montré que les anomalies du flagelle étaient sous-estimées car mal vues par insuffisance de contraste.

Devant l'importante variation des grilles de lecture, même si elles se réferent pour une majorité à la grille de David, il existe un grand besoin de normalisation. Ainsi le groupe de travail propose une synthèse entre la classification de David avec celle proposée par l'OMS de 1994.

Dans cette classification sont notés :

- les anomalies de forme et de taille de la tête

- les anomalies du col et de la pièce intermédiaire

- les anomalies du flagelle

- les restes cytoplasmiques.

Cette classification n'étant pas en contradiction avec la classification de David, le groupe propose une nouvelle présentation qui associe les deux classifications (Figure 2). 
A - FORMES TYPIQUES :

B - FORMES ATYPIQUES :

1. TETE

Allongée (a)

\begin{tabular}{lcc}
\hline Amincie (b) & $\square$ \\
\hline Microcéphale (c) & $\square$ \\
\hline Macrocéphale(d) & \\
\hline
\end{tabular}

Têtes multiples (e)

Base anormale (f)

Acrosome absent ou anormal (g)

2. RESTE CYTOPLASMIQUE (h)

3. PIECE INTERMEDIAIRE

Grêle (i)

Angulé (j)

4. FLAGELLE

Absent (k)

\begin{tabular}{lll}
\hline Ecourté $(\mathbf{l})$ & $\square$ \\
\hline Calibre irrégulier $(\mathrm{m})$ & $\square$ \\
\hline Enroulé (o) & $\square$ \\
\hline Multiple (p) & $\square$ \\
\hline
\end{tabular}

I.A.M.

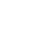

Figure 2 : Modèle de grille de rendu d'analyse du spermocytogramme proposé par le Groupe de Travail :"Assurance de qualité ren Biologie de la Reproduction“. 
Le calcul de l'Indice d'Anomalies Multiples (IAM) est le paramètre qui, lors des contrôles des qualité devient le plus rapidement précis, puis exact (voir l'article de Bourguignat et Coll.), il est dommage que dans de nombreux laboratoires il ne soit pas calculé. Cet indice est le rapport entre le nombre d'anomalies observées et le nombre de spermatozoïdes anormaux.

Pour renforcer la puissance diagnostique de l'indice il faut savoir qu'il est possible d'observer jusqu'à 4 anomalies par spermatozoïde.

\section{Cellules rondes}

Les cellules rondes constituent un élément important du spermogramme lui conférant une puissance diagnostique supplémentaire. Quand il est possible de mettre en évidence des polynucléaires, l'existence d'un processus infectieux en cours d'évolution peut être affirmée.

L'utilisation de la technique de recherche de la peroxydase apporte un élément diagnostique précieux dont beaucoup de laboratoires se privent. Cette technique relativement facile est à la portée de tous.

L'étude morphologique que de nombreux laboratoires utilisent est un bon complément mais ne permet pas toujours de faire des diagnostics, certains d'autant que les cellules retrouvées dans l'éjaculat sont souvent en mauvais état de conservation.

\section{CONCLUSIONS}

L'enquête réalisée auprès de 214 laboratoires révèle une importante diversité méthodologique et justifie un effort de normalisation.

Cet effort de normalisation doit viser dans un premier temps :

- à travailler à un température de $37^{\circ} \mathrm{C}$,

- à évaluer la viscosité après une incubation de 30 minutes,
- à réaliser une numération après une dilution adaptée à la concentration évaluée et associée à la lecture de deux grilles.

- à évaluer la mobilité au microscope à contraste de phase équipé d'une platine chauffante, à partir d'une préparation à goutte de sperme calibrée en fonction de la taille de la lamelle, en utilisant la classification de l'OMS,

- à utiliser, pour le spermocytogramme, la classification proposée par le groupe de travail,

- à calculer l'Indice d'Anomalies Multiples,

- à mettre en évidence les polynucléaires par la réaction histoenzymatique de la peroxydase.

\section{OUVRAGE DE REFERENCE}

MANUEl DE LABORATOIRE DE L'OMS. Analyse du sperme humain et de l'interaction des spermatozoïdes avec le mucus cervical. Traduction de Jacques AUGER et Pierre JOUANNET. Les Editions INSERM 1993.

\begin{abstract}
The spermiogram in french biological laboratories

A. Clavert, A. Bourguignat, J.P. Siest, G. FERARD

An extensive investigation was performed by numerous public as well private biological laboratories. 214 answers were collected. Most of them corresponded to laboratories analysing less than 5 spermiograms per week. Only 74 of them can be considered as specialised in this field.
\end{abstract}

Among the questions, those conserning sperm conservation, viscosity and motylity evaluation, spermatozoa count, rate of teratospermia, study of rund cells revealed marked differences in the routine practice. These differences can affect the diagnostic value of the spermiogram. 
To reach ab harmonisation of the results, some recommendations are proposed by the working group. First, treament and microscopic examinations should be carried out at $37^{\circ} \mathrm{C}$; WHO recommendation should be followed for the motility and teratospermia rate evaluation. A common format is also proposed to present results conserning the spermiocytogram, contributing to improve the standardisation.

Key words : Spermiogram, Practices, Procedures. 